\title{
Influência dos níveis séricos de IGF-I e de testosterona sobre o perfil lipídico e glicêmico em homens acromegálicos
}

\author{
Influence of serum IGF-I and testosterone on lipid \\ and glycemic profile in acromegalic men
}

Patrícia R. L. Figueiredo', Manoel R. A. Martins' Renan M. Montenegro', Mônica F. Albano', Renan M. Montenegro Jr.'

'Serviço de Endocrinologia e Diabetes, Hospital Universitário Walter Cantídio, Universidade Federal do Ceará (HUWCUFC), Fortaleza, CE, Brasil
Correspondência para:

Renan M. Montenegro Jr. Faculdade de Medicina,

Universidade Federal do Ceará

Rua Prof. Costa Mendes, 1.608,

$5^{\circ}$ andar

60416-200 - Fortaleza, CE, Brasil

renanjr@ufc.br

Recebido em 8/Ago/2009 Aceito em 13/Nov/2009

\begin{abstract}
RESUMO
Objetivos: Investigar a influência do IGF-I e testosterona sobre o perfil lipídico e glicêmico em homens acromegálicos. Métodos: Foram avaliados 15 homens acromegálicos. Resultados: Os pacientes hipogonádicos apresentaram HDL-c mais baixo e triglicérides, LDL-c, glicemia, GH e IGF-I mais elevados. Os valores de IGF-I correlacionaram-se inversamente com HDL-c $(r=-0,57, p=0,04)$ e diretamente com triglicerídeos $(r=0,62, p=0,01)$ e glicemia $(r=0,66, p$ $=0,008)$, enquanto a testosterona correlacionou-se diretamente com HDL-c $(r=0,52, p=0,05)$ e inversamente com triglicerídeos $(r=-0,57, p=0,02)$; $L L(r=-0,53, p=0,04)$ e glicemia $(r=-0,54, p=0,03)$. O IGF-I e a testosterona correlacionaram-se inversamente $(r=-0,585, p=$ 0,028 ). Conclusão: Estes resultados sugerem que a intervenção mais importante na redução do risco cardiovascular nesses pacientes é o controle da atividade da doença. Arq Bras Endocrinol Metab. 2009;53(8):1026-32

Descritores

Acromegalia; hipogonadismo; testosterona; IGF-I; lípides; glicemia; síndrome metabólica; risco cardiovascular
\end{abstract}

\begin{abstract}
Objectives: To evaluate the influence of IGF-I and testosterone on the lipid profile and glycemia in acromegalic men. Methods: Fifteen acromegalic men were studied. Results: The hypogonadic patients presented lower HDL-c and higher tryglicerides, LDL-c, glycemia, GH and IGF-I. Serum IGF-I was inversely correlated with HDL-c $(r=-0.57, p=0.04)$ and directly with TG $(r=0.62, p=0.01)$ and glycemia $(r=0.66, p=0.008)$, whereas serum testosterone correlated directly with HDL-c $(r$ $=0.52, p=0.05)$ and inversely with TG $(r=-0.57, p=0.02)$, LDL-c $(r=-0.53, p=0.04)$ and fasting blood glucose $(r=-0.54, p=0.03)$. IGF-I and testosterone were inversely correlated $(r=-0.585$, $p=0.028$ ). Conclusion: These results suggest that the most important intervention in reducing cardiovascular risk in these patients is to control the activity of the disease. Arq Bras Endocrinol Metab. 2009;53(8):1026-32
\end{abstract}

Keywords

Acromegaly; hypogonadism; testosterone; IGF-I; lipids; glycemia; metabolic syndrome; cardiovascular risk

\section{INTRODUÇ̃̃O}

A acromegalia é causada pela hipersecreção crônica - do hormônio do crescimento $(\mathrm{GH})$, levando a um aumento da produção periférica do fator de crescimento semelhante à insulina tipo 1 (IGF-I) (1-2). Os pacientes acromegálicos têm uma taxa de mortalidade aumentada em cerca de duas vezes à da população geral em conse- quência, principalmente, das complicações cardiovasculares (3-4).

A síndrome metabólica (SM) é um importante fator de risco independente para o desenvolvimento de diabetes tipo 2 (DM2) e doenças cardiovasculares (DCV) na população geral, associada principalmente ao aumento da resistência à insulina (RI), deposição central de gor- 
dura, elevação da pressão arterial e dislipidemia (5-6). $\mathrm{Na}$ acromegalia, tanto o aumento do GH quanto a deficiência androgênica podem contribuir para alterações no perfil metabólico.

$\mathrm{O}$ excesso de $\mathrm{GH}$ pode induzir alterações no metabolismo dos carboidratos e dos lipídios devido ao aumento da resistência à insulina, com redução do consumo de glicose pelos músculos esqueléticos, e ao aumento da lipólise e da gliconeogênese hepática $(2,7)$. A intolerância à glicose ou o diabetes melito $(\mathrm{DM})$ estão presentes na maioria dos pacientes acromegálicos (8). O GH exerce um efeito lipolítico global, induzindo a hidrólise de triglicerídeos a ácidos graxos livres e glicerol (9). As alterações no perfil lipídico vistas em acromegálicos são caracterizadas por redução do HDLcolesterol (HDL-c), presença de partículas de LDLcolesterol (LDL-c) mais densas e pequenas e aumento da lipoproteína-a (LP-a) $(4,10)$.

A deficiência androgênica é outro fator que pode induzir a SM em pacientes acromegálicos. O hipogonadismo hipogonadotrófico é bastante frequente em homens acromegálicos, mesmo em pacientes jovens (11), em decorrência da compressão tumoral, da hiperprolactinemia, do tratamento cirúrgico ou da radioterapia. Níveis séricos baixos de testosterona (T) e da proteína ligadora dos hormônios sexuais (SHBG) são fatores de risco para SM (12). A obesidade abdominal está fortemente relacionada à SM e à RI e tem sido associada a níveis mais baixos de T (13). Hipertensão arterial, níveis baixos de HDL-c e hipertrigliceridemia também têm sido associados ao hipogonadismo (14).

Não está claro se as alterações no perfil metabólico em pacientes acromegálicos estão associadas principalmente à atividade da doença ou ao hipogonadismo. Neste estudo, foi investigada a influência dos valores de IGF-1 e de testosterona sobre o perfil lipídico e glicêmico em homens acromegálicos.

\section{MÉTODOS}

Avaliaram-se as fichas clínicas de 22 pacientes do sexo masculino com diagnóstico de acromegalia entre 1992 e 2009. Foram incluídos para análise 15 pacientes que tinham dosados LH, FSH, testosterona total, perfil lipídico e glicemia de jejum na última consulta. Sete pacientes foram excluídos por não apresentarem dosagem de testosterona na última avaliação.

Avaliaram-se os pacientes conforme o estado gonadal e a atividade da doença. Os pacientes foram ini- cialmente divididos em dois grupos: hipogonádicos (HIPO) e eugonádicos (EU). Foram considerados hipogonádicos os pacientes com níveis de testosterona total inferior a 1,95 ng/mL. Em seguida, compararamse os mesmos pacientes de acordo com a atividade da doença, controlados (IGF-l normal para idade e sexo) ou em atividade (IGF-1 elevada).

As variáveis comparadas entre os grupos foram glicemia de jejum, colesterol T (CT), HDL-c, LDL-c, triglicérides (TG), índice de massa corporal (IMC), circunferência abdominal (CA), pressão arterial sistólica (PAS), pressão arterial diastólica (PAD), IGF-1, GH, presença de síndrome metabólica, tipos de tratamento (cirurgia, radioterapia ou medicamentoso), tamanho tumoral (macro ou microadenoma).

\section{Medidas antropométricas}

O IMC foi calculado pela razão entre o peso (em quilogramas) e o quadrado da altura (em metros) $\left(\mathrm{kg} / \mathrm{m}^{2}\right)$. A circunferência abdominal foi aferida à meia distância entre a face inferior da última costela e a porção superior da crista ilíaca.

\section{Definição de síndrome metabólica}

A presença de SM foi definida segundo os critérios da International Diabetes Federation (IDF), na presença de obesidade central (circunferência abdominal $>94 \mathrm{~cm}$ ), associada a duas ou mais das seguintes condições: triglicerídeos $\geq 150 \mathrm{mg} / \mathrm{dL}$; HDL-c $<40 \mathrm{mg} / \mathrm{dL}$; pressão arterial sistólica $\geq 130 \mathrm{mmHg}$ ou diastólica $\geq 85$ $\mathrm{mmHg}$; glicemia em jejum $\geq 100 \mathrm{mg} / \mathrm{dL}$ (15).

\section{Determinações laboratoriais}

O método utilizado para dosagem de GH foi quimioluminescência (Siemens Medical Solutions, Los Angeles, CA, com o analisador IMMULITE 1000), direcionado especificamente para detecção da isoforma de GH com peso molecular de $22 \mathrm{kDa}$. A sensibilidade do método é de $0,01 \mathrm{ng} / \mathrm{mL}(0,026 \mathrm{mU} / \mathrm{L})$. Os coeficientes de variação inter e intraensaio são de 5,5\%-6,2\% e 5,3\%$6,5 \%$, respectivamente.

Na dosagem de IGF-I, o método utilizado foi imunorradiométrico com extração prévia das proteínas carreadoras com etanol (DSL - Diagnostic Systems Laboratories, Inc., Webster, TX). A sensibilidade do método é de $0,80 \mathrm{ng} / \mathrm{mL}$. O coeficiente de variação interensaio é de $8,2 \%, 1,5 \%$ e $3,7 \%$ para baixo, médio e alto ponto da curva padrão, respectivamente. O coe- 
ficiente de variação intraensaio é de $3,4 \%, 3,0 \%$ e 1,5\% para baixo, médio e alto ponto da curva padrão, respectivamente. Os valores de referência são indicados por sexo e idade.

Para dosagem de LH, FSH, prolactina (PRL); T4L; testosterona $(\mathrm{T})$ foi utilizada a técnica de ensaio imunoenzimático de micropartículas (Abbott $\mathrm{AxSYM}^{\circledR}$ System). As sensibilidades dos métodos são: LH (0,5 $\mu \mathrm{UI} / \mathrm{mL}) ; \mathrm{FSH}(0,37 \mathrm{mUI} / \mathrm{mL}) ; \mathrm{PRL}(0,6 \mathrm{ng} / \mathrm{mL})$; T4L $(0,4 \mathrm{ng} / \mathrm{mL}) ; \mathrm{T}(0,1 \mathrm{ng} / \mathrm{mL})$. Os coeficientes de variação intraensaio são: $\mathrm{LH}(4,15 \%$ a $6,71 \%) ; \mathrm{FSH}$ $(3,65 \%$ a $5,94 \%)$; PRL $(2,81 \%$ a $4,13 \%)$; T4L $(2,1 \%$ a $5,96 \%)$; T $(3,9 \%$ a $8,3 \%)$. Os coeficientes de variação interensaio são: LH (0,0 a 6,39\%); PRL (0,0 a 2,76\%); T4L $(0,0$ a 4,32\%). Os valores de referência são: $\mathrm{LH}$ (2 a $12 \mu \mathrm{UI} / \mathrm{mL})$; FSH (1,13 a 12,51 $\mu \mathrm{UI} / \mathrm{mL})$; PRL $(3,28$ a $19,68 \mathrm{ng} / \mathrm{mL})$; T4L $(0,71$ a $1,85 \mathrm{ng} / \mathrm{mL})$ e T $(1,95$ a $11,38 \mathrm{ng} / \mathrm{mL})$.

O TSH sérico foi dosado por meio do ensaio AxSYM $3^{\text {rd }}$ Generation TSH, imunoensaio enzimático por micropartículas (MEIA, Abbott AxSYM ${ }^{\circledast}$ System). A sensibilidade do método é de $0,006 \mu \mathrm{UI} / \mathrm{mL}$. O coeficiente de variação intraensaio é de $2,9 \%$ a $11,0 \%$. O valor de referência é de 0,47 a $4,64 \mu \mathrm{UI} / \mathrm{mL}$.

O método utilizado para dosagem da glicose foi cinético UV hexoquinase com o analisador Dimension RXL Dade Behring. A sensibilidade do método é de $0,80 \mathrm{mg} / \mathrm{dL}$. Os coeficientes de variação inter e intraensaio são de $1,4 \%$ e $2,82 \%$, respectivamente. $O$ valor de referência é de 70-99 mg/dL.

\section{Análise estatística}

A análise estatística foi realizada com teste $t$ de Student ou Mann Whitney, conforme os dados fossem paramétricos ou não paramétricos, respectivamente, para verificar diferenças entre duas variáveis, e o teste exato de Fischer para comparação entre frequências. A associação linear entre duas variáveis foi feita pelo coeficiente de correlação de Pearson.

\section{RESULTADOS}

\section{Características dos pacientes}

Quinze pacientes foram incluídos para análise. Cinco pacientes tinham hipogonadismo hipogonadotrófico (grupo HIPO) e 10 pacientes eram eugonádicos (grupo EU). A média de idade nos dois grupos foi semelhante (HIPO 48,4 \pm 12,3 anos, intervalo: 36-64 anos;
EU 49,7 \pm 13,4 anos, intervalo: $30-66$ anos, $\mathrm{p}=0,86$ ). Nenhum paciente apresentava hipotireoidismo ou hiperprolactinemia.

\section{Comparações de acordo com 0 estado gonadal}

Quando comparados os acromegálicos eugonádicos com os acromegálicos hipogonádicos, observou-se que estes últimos apresentaram níveis séricos mais baixos de HDL-c e mais elevados de triglicérides, LDL-c e glicemia de jejum em relação aos primeiros (Tabela 1). Os níveis de colesterol total tenderam a ser mais elevados nos hipogonádicos (Tabela 1 ). A prevalência de síndrome metabólica tendeu a ser mais elevada nos hipogonádicos $(80 \%$ vs. $20 \%, \mathrm{p}=0,086)$. Não houve diferenças entre os grupos HIPO e EU com relação ao tratamento (realização de cirurgia, radioterapia, uso de octreotida LAR e cabergolina ou terapia primária) ou tamanho do tumor ao diagnóstico (macro ou microadenoma). Ao mesmo tempo, os pacientes hipogonádicos apresentavam valores mais elevados de GH e IGF-I (Tabela 1).

\section{Comparações de acordo com a atividade da acromegalia}

Os pacientes com doença em atividade apresentavam níveis mais elevados de glicemia de jejum e de triglicérides que aqueles com doença controlada (Tabela 2 ).

\begin{tabular}{|c|c|c|c|}
\hline & HIPO & EU & $\mathbf{p}$ \\
\hline Idade & $48,4 \pm 12,3$ & $49,7 \pm 13,4$ & 0,86 \\
\hline $\mathrm{GH}(\mathrm{ng} / \mathrm{mL})$ & $4,69 \pm 3,92$ & $1,3 \pm 1,13$ & 0,03 \\
\hline IGF1 (ng/mL) & $573,7 \pm 507,9$ & $181 \pm 94,3$ & 0,035 \\
\hline LH (mUl/mL) & $2,38 \pm 1,47$ & $3,88 \pm 2,02$ & 0,016 \\
\hline $\mathrm{FSH}(\mathrm{mUl} / \mathrm{mL})$ & $4,23 \pm 1,98$ & $5,6 \pm 2,26$ & 0,27 \\
\hline Testosterona (ng/mL) & $1,39 \pm 0,75$ & $4,25 \pm 1,35$ & 0,0008 \\
\hline GJ (mg/dL) & $113,4 \pm 18,5$ & $91,9 \pm 9$ & 0,03 \\
\hline IMC (kg/m²) & $31,4 \pm 1,7$ & $28,2 \pm 2,7$ & 0,37 \\
\hline $\mathrm{HDL}(\mathrm{mg} / \mathrm{dL})$ & $34,8 \pm 9,2$ & $53,4 \pm 17,3$ & 0,047 \\
\hline LDL (mg/dL) & $114,8 \pm 37,7$ & $81,6 \pm 18,2$ & 0,04 \\
\hline CT (mg/dL) & $188,4 \pm 43,6$ & $152,9 \pm 23,9$ & 0,069 \\
\hline $\mathrm{TG}(\mathrm{mg} / \mathrm{dL})$ & $193,6 \pm 60,7$ & $101,1 \pm 50,5$ & 0,0078 \\
\hline $\mathrm{CA}(\mathrm{cm})$ & $103,4 \pm 7,7$ & $96,7 \pm 10$ & 0,22 \\
\hline PAS (mmHg) & $140 \pm 20$ & $140,5 \pm 18,3$ & 0,96 \\
\hline PAD (mmHg) & $97 \pm 17,9$ & $90,6 \pm 17,6$ & 0,52 \\
\hline
\end{tabular}

GJ: glicemia de jejum; IMC: índice de massa corporal; CT: colesterol total; TG: triglicerídeos; CA: circunferência abdominal; PAS: pressão arterial sistólica; PAD: pressão arterial diastólica. 


\section{Correlações entre IGF-I ou testosterona e as variáveis estudadas}

Os valores de IGF-I correlacionaram-se inversamente com níveis de HDL-c $(\mathrm{r}=-0,57, \mathrm{p}=0,04)$ e diretamente com os níveis de TG $(\mathrm{r}=0,62, \mathrm{p}=0,01)$ e de glicemia de jejum $(\mathrm{r}=0,66, \mathrm{p}=0,008)$ (Tabela 3$)$. Os níveis séricos de testosterona correlacionaram-se diretamente com níveis de HDL-c $(\mathrm{r}=0,52, \mathrm{p}=0,05)$ e inversamente com os níveis de TG $(\mathrm{r}=-0,57, \mathrm{p}=$ $0,02) ; \operatorname{LDL}(-0,53, \mathrm{p}=0,04)$ e de glicemia de jejum $(\mathrm{r}$ $=-0,54, \mathrm{p}=0,03)$ (Tabela 4$)$. Os níveis séricos de $\mathrm{GH}$ não se correlacionaram com as variáveis estudadas. Os valores de IGF-I e testosterona correlacionaram-se de maneira inversa $(\mathrm{r}=-0,585, \mathrm{p}=0,028)$.

\begin{tabular}{lccc}
\hline $\begin{array}{l}\text { Tabela 2. Características clínicas e laboratoriais dos pacientes em atividade da } \\
\text { doença }\end{array}$ \\
\hline Doença em atividade & Em atividade & Controlada & p \\
\hline Idade & $47,0 \pm 5,8$ & $50,40 \pm 4,097$ & 0,64 \\
GH (ng/mL) & $13,9 \pm 8,80$ & $1,13 \pm 0,312$ & 0,042 \\
IGF1 (ng/mL) & $659,2 \pm 160,0$ & $145,3 \pm 9,63$ & 0,0008 \\
LH (mUl/mL) & $2,038 \pm 0,47$ & $3,61 \pm 0,29$ & 0,2 \\
FSH (mUl/mL) & $4,48 \pm 0,9$ & $5,41 \pm 0,71$ & 0,46 \\
Testosterona (ng/mL) & $2,26 \pm 0,79$ & $3,97 \pm 0,48$ & 0,007 \\
GJ (mg/dL) & $110,6 \pm 9,87$ & $85,29 \pm 7,0$ & 0,05 \\
IMC (kg/m²) & $30,16 \pm 0,67$ & $29,14 \pm 1,21$ & 0,53 \\
HDL (mg/dL) & $36,80 \pm 5,49$ & $52,33 \pm 5,86$ & 0,10 \\
LDL (mg/dL) & $93,60 \pm 9,16$ & $93,40 \pm 11,89$ & 0,99 \\
CT (mg/dL) & $166,2 \pm 14,57$ & $165,2 \pm 12,92$ & 0,78 \\
TG (mg/dL) & $178,6 \pm 36,82$ & $98,30 \pm 12,31$ & 0,02 \\
CA (cm) & $99,75 \pm 2,76$ & $98,63 \pm 4,11$ & 0,25 \\
PAS (mmHg) & $138,0 \pm 9,6$ & $135,5 \pm 8,2$ & 0,85 \\
PAD (mmHg) & $94,2 \pm 9,03$ & $93,33 \pm 5,77$ & 0,93 \\
\hline
\end{tabular}

GJ: glicemia de jejum; IMC: índice de massa corporal; CT: colesterol total; TG: triglicerídeos; CA: circunferência abdominal; PAS: pressão arterial sistólica; PAD: pressão arterial diastólica.

\begin{tabular}{|c|c|c|c|}
\hline & $\mathbf{R}$ & p & $\mathbf{r}^{2}$ \\
\hline GJ (mg/dL) & 0,66 & 0,0088 & 0,44 \\
\hline IMC (kg/m²) & 0,17 & 0,55 & 0,29 \\
\hline $\mathrm{HDL}(\mathrm{mg} / \mathrm{dL})$ & $-0,57$ & 0,04 & 0,32 \\
\hline LDL (mg/dL) & $-0,051$ & 0,86 & 0,002 \\
\hline CT (mg/dL) & $-0,04$ & 0,88 & 0,0018 \\
\hline $\mathrm{TG}(\mathrm{mg} / \mathrm{dL})$ & 0,628 & 0,016 & 0,394 \\
\hline $\mathrm{CA}(\mathrm{cm})$ & 0,042 & 0,88 & 0,0018 \\
\hline PAS (mmHg) & 0,23 & 0,42 & 0,05 \\
\hline PAD (mmHg) & 0,36 & 0,199 & 0,133 \\
\hline
\end{tabular}

GJ: glicemia de jejum; IMC: índice de massa corporal; CT: colesterol total; TG: triglicerídeos; CA: circunferência abdominal; PAS: pressão arterial sistólica; PAD: pressão arterial diastólica.

\begin{tabular}{lccc}
\hline \multicolumn{4}{c}{ Tabela 4. Correlação entre níveis séricos de T e os componentes da SM } \\
\hline & $\mathbf{r}$ & $\mathbf{p}$ & $\mathbf{r}^{2}$ \\
\hline GJ (mg/dL) & $-0,54$ & 0,03 & 0,29 \\
IMC (kg/m²) & $-0,26$ & 0,33 & 0,07 \\
HDL (mg/dL) & 0,52 & 0,05 & 0,27 \\
LDL (mg/dL) & $-0,53$ & 0,04 & 0,28 \\
CT (mg/dL) & $-0,50$ & 0,06 & 0,25 \\
TG (mg/dL) & $-0,57$ & 0,02 & 0,33 \\
CA (cm) & $-0,11$ & 0,69 & 0,01 \\
PAS (mmHg) & 0,10 & 0,71 & 0,01 \\
PAD (mmHg) & 0,03 & 0,90 & 0,001 \\
\hline
\end{tabular}

GJ: glicemia de jejum; IMC: índice de massa corporal; CT: colesterol total; TG: triglicerídeos; CA: circunferência abdominal; PAS: pressão arterial sistólica; PAD: pressão arterial diastólica.

\section{DISCUSSÃO}

$\mathrm{Na}$ literatura, os dados em relação aos efeitos do hipogonadismo no perfil metabólico de acromegálicos são escassos. Na população geral, a deficiência androgênica pode contribuir para o surgimento de alterações no perfil glicídico e lipídico(16). Em obesos, níveis séricos de testosterona se correlacionam inversamente com a obesidade visceral, a síndrome metabólica, a resistência à insulina (17), a hipertensão arterial, os níveis baixos de HDL-c e a hipertrigliceridemia (18). Em homens, a interação entre os níveis de testosterona e o metabolismo dos lípides e o risco cardiovascular é complexa e não está completamente estabelecida (19). Na presente amostra de homens acromegálicos, os hipogonádicos apresentaram níveis séricos de HDL-c mais baixos e de triglicérides, LDL-c e glicemia de jejum mais elevados, de forma semelhante aos hipogonádicos da população geral. A prevalência de síndrome metabólica tendeu a ser mais elevada nos hipogonádicos em comparação com os eugonádicos, provavelmente em razão do tamanho dessa amostra.

O hipogonadismo é uma consequência potencial da acromegalia, devido à compressão tumoral, à hiperprolactinemia, ao tratamento cirúrgico ou à radioterapia. (20). Além disso, os níveis elevados de GH podem induzir diretamente o hipogonadismo, e o controle da doença com octreotida aumenta os níveis de andróge$\operatorname{nos}(21)$.

Os efeitos da testosterona sobre os valores de GH/ IGF-I podem ser diretos ou indiretos, via estradiol $(22,23)$. Diretamente, a testosterona amplifica a pulsatilidade do GH e a ativação do eixo GH/IGF-I em homens hipogonádicos, mas não em eugonádicos (23). No entanto, a testosterona não aumenta diretamente 
a síntese hepática de IGF-I induzida pelo GH. O aumento do estradiol, convertido a partir da testosterona, promove a secreção de GH sem elevar as concentrações de IGF-I (24). Os efeitos dos estrógenos nas concentrações de IGF-I são devidos à inibição da síntese hepática de IGF-I (25) e à potencialização do feedback IGF-I-dependente sobre GH (26). No presente estudo, os pacientes hipogonádicos apresentavam valores mais elevados de GH e IGF-I. Entretanto, os valores de IGF-I e testosterona correlacionaram-se de maneira inversa, o que poderia ser explicado pelos efeitos diretos do GH/IGF-1 nos níveis de testosterona. No entanto, só é possível definir uma relação causal em um estudo longitudinal.

Em homens hipogonádicos, a terapia de reposição com T melhora a energia, a composição corporal e outras anormalidades (27). Com relação ao perfil metabólico, a administração de andrógenos exógenos reduz os níveis de HDL em adultos jovens saudáveis e hipogonádicos (28), porém esse efeito não é visto em homens idosos (29). Em contraste, outros estudos transversais, em homens adultos, têm mostrado o aumento do HDL-c com elevação dos níveis de testosterona (30). A reposição de testosterona em homens hipogonádicos com diabetes tipo 2 pode melhorar a glicemia de jejum, insulina de jejum, hemoglobina glicada (HbAlc) e peso (31). Não há dados na literatura em relação aos efeitos da reposição de T no perfil metabólico de pacientes acromegálicos.

$\mathrm{O}$ excesso de $\mathrm{GH}$ pode causar RI com hiperinsulinemia compensatória (7). Se a RI for maior que a capacidade de produção compensatória de insulina, pode ocorrer intolerância à glicose $(50 \%$ dos pacientes $)$ ou $\mathrm{DM}$ ( $10 \%$ a $25 \%$ dos pacientes) (2). A acromegalia também está associada à dislipidemia, porém os estudos para avaliar o metabolismo lipídico nos acromegálicos são conflitantes. Os níveis de colesterol total foram descritos como normais, aumentados ou diminuídos (4). As partículas de LDL pequenas e densas estavam elevadas na maioria dos estudos $(4,10)$. O nível de HDL-c foi descrito como inalterado (32) ou diminuído (33). A incidência de hipertrigliceridemia em acromegálicos é similar à da população geral (4), porém a hipertrigliceridemia tipo IV é três vezes maior na população acromegálica (10). As alterações dos lipídeos nos acromegálicos são mais evidentes nos pacientes com alterações concomitantes no metabolismo glicêmico (4). No presente estudo, os pacientes acromegálicos em atividade da doença apresentaram níveis mais elevados de gli- cemia de jejum e de triglicérides que aqueles com doença controlada. Não houve diferenças entre os níveis de HDL-c, possivelmente pelo tamanho da amostra. Os níveis de LDL-c não foram influenciados pela atividade da doença, possivelmente porque a alteração mais comum descrita ocorre nas partículas de LDL pequenas e mais densas $(4,10)$ e não no LDL total.

As alterações do perfil metabólico são modificadas após a normalização dos valores de GH/IGF-I (34). A cura cirúrgica da acromegalia está associada à melhora da tolerância à glicose e do diabetes (35). A resposta do metabolismo da glicose ao tratamento com octreotida é resultado de um balanço entre seus efeitos inibitórios sobre a secreção pancreática de insulina e glucagon e da melhora da RI pela diminuição do GH (36). Em geral, os análogos da somatostatina melhoram o perfil lipídico, mas não modificam as partículas de LDL pequenas e densas, que são mais aterogênicas (33). $\mathrm{O}$ pegvisomanto, antagonista do receptor de $\mathrm{GH}$, reduz os níveis basais de insulina, glicose, colesterol total e de triglicerídeos, em decorrência da diminuição da resistência insulínica $(4,37)$.

Não está claro se as alterações no perfil metabólico em pacientes acromegálicos estão associadas principalmente à atividade da doença ou ao hipogonadismo. Na presente amostra, os valores de IGF-I correlacionaram-se inversamente com HDL-c e diretamente com os níveis de triglicérides e de glicemia de jejum, enquanto os níveis de testosterona correlacionaram-se diretamente com níveis de HDL-c e inversamente com os níveis de TG, LDL-c e de glicemia de jejum. Esses resultados sugerem que tanto a atividade de doença quanto o estado gonadal se correlacionam com componentes da síndrome metabólica. A correlação inversa entre IGF-I e testosterona, no entanto, sugere que o determinante principal de síndrome metabólica em acromegálicos é o IGF-I.

O presente estudo apresenta algumas limitações. A amostra pequena de pacientes não permitiu a avaliação de possíveis fatores confundidores que poderiam ter contribuído para os resultados. Além disso, o desenho transversal do estudo não permite estabelecer uma relação de causa e efeito. A dosagem de testosterona livre, e não de total como foi realizada, poderia ser um parâmetro mais acurado do estado gonadal.

Esses resultados sugerem que a intervenção mais importante na redução do risco cardiovascular nesses pacientes é o controle da atividade da doença. São necessários estudos prospectivos, longitudinais, avaliando as repercussões do estado gonadal e da atividade 
da doença no perfil metabólico e na determinação da síndrome metabólica em acromegálicos. Além disso, é desconhecido se a reposição androgênica nos homens acromegálicos hipogonádicos pode melhorar o seu perfil metabólico, modificando os componentes da SM e diminuindo o risco cardiovascular desses pacientes.

Agradecimentos: os autores agradecem a Dra. Nazaré de Moura Rocha, farmacêutica do Hospital Universitário Walter Cantídio da Universidade Federal do Ceará, pela realização das análises bioquímicas e dosagens hormonais.

Declaração: os autores declaram não haver conflitos de interesse científico neste estudo.

\section{REFERÊNCIAS}

1. Le Roith $D$, Scavo L, Butler A. What is the role of circulating IGF-I? Trends Endocrinol Metab. 2001;12:48-52.

2. Melmed S. Acromegaly. N Engl J Med. 2006;355:2558-73.

3. Holdaway IM, Rajassorya RC, Gamble GD. Factors influencing mortality in acromegaly. J Clin Endocrinol Metab. 2004;89:667-74.

4. Colao A, Ferone D, Marzullo P, Lombardi G. Systemic complications of acromegaly: epidemiology, pathogenesis, and management. Endocrine Reviews. 2004;25(1):102-52.

5. Lakka HM, Laaksonen DE, Lakka TA, Kumpusalo E, Tuomilehto J, Salonen JT.The metabolic syndrome and total and cardiovascular disease mortality in middle-aged men. JAMA. 2002;288:2709-16.

6. Haffner S, Taegmeyer H. Epidemic obesity and the metabolic syndrome. Circulation. 2003;108:1541-5.

7. Moller N, Schmitz O, Joorgensen JO, Astrup J, Back JF, Christensen $\mathrm{SE}$, et al. Basal and insulin-stimulated substrate metabolism in patients with active acromegaly before and after adenomectomy. J Clin Endocrinol Metab. 1992;74:1012-9.

8. Colao A, Baldelli R, Marzullo P, Ferretti E, Ferono D, Gargiulo P, et al. Systemic hypertension and impaired glucose tolerance are independently correlated to the severity of the acromegalic cardiomyopathy. J Clin Endocrinol Metab. 2000;85:193-9.

9. Wildbrett J, Hanefeld M, Fucker K, Pinzer T, Bergmann S, Siegert $G$, et al. Anomalies of lipoprotein pattern and fibrinolysisin acromegalic patients: relation to growth hormone levels and insulinlike growth factor I. Exp Clin Endocrinol Diabetes. 1997;105:331-5.

10. Nikkila EA, Pelkonen R. Serum lipids in acromegaly. Metabolism. 1975;24:829-38.

11. Katznelson L, Kleinberg D, Vance ML, Stravou S, Pulaski KJ, Schoenfeld DA, et al. Hypogonadism in patients with acromegaly: data from the multi-centre acromegaly registry pilot study. Clin Endocrinol. 2001;54:183-8.

12. Kupelian V, Page ST, Araujo AB, Travison TG, Bremner WJ, Mckinlay JB. Low sex hormone-binding globulin, total testosterone, and symptomatic androgen deficiency are associated with development of the metabolic syndrome in nonobese men. J Clin Endocrinol Metab. 2006;91:843-50.

13. Blouin K, Despres JP, Couillard C, Tremblay A, Prud'homme D, Bouchard $\mathrm{C}$, et al. Contribution of age and declining androgen levels to features of the metabolic syndrome in men. Metabolism. 2005;54:1034-40.

14. Seidell JC, Bjomtorp P, Sjostrom L, Kvist H, Sannerstedet R. Visceral fat accumulation in men is positively associated with insulin,glucose and C-peptide levels, but negatively with testosteronelevels. Metabolism. 1990;39:897-901.
15. International Diabetes Federation: The IDF consensus worldwide definition of the metabolic syndrome. April 14, 2005. Disponível em: http://www.idf.org/webdata/docs/Metac_syndrome_def.pdf.

16. Guay AT. The emerging link between hypogonadism and metabolic syndrome. J Andrology. 2009;30(4):370-6.

17. Seidell JC, Bjomtorp P, Sjostrom L, Kvist H, Sannerstedet R. Visceral fat accumulation in men is positively associated with insulin, glucose and C-peptide levels, but negatively with testosterone levels. Metabolism. 1990;39:897-901.

18. Haider A, Yassin A, Saad F, Shabsigh R. Effects of androgen deprivation on glycemic control and on cardiovascular biochemical risk factors in men with advanced prostate cancer with diabetes. Aging Male. 2007;10:189-96.

19. Cohen PG. Aromatase, adiposity, aging and disease. The hypogonadal-metabolic-atherogenic-disease and aging conection. Med Hypothesis. 2001;56:702-8.

20. Katznelson L, Kleinberg D, Vance ML, Stravou S, Pulaski KJ, Schoenfeld DA, et al. Hypogonadism in patients with acromegaly: data from the multi-centre acromegaly registry pilot study. Clinic levels improves gonadal function and sperm parameters in men with acromeal. Endocrinology. 2001;54:183-8.

21. Colao A, Rosa M, Pivonello R, Balestrieri A, Cappabianca P, Di Sarno A, et al. Short-term suppression of GH and IGF-I galy. J Clin Endocrinol Metab. 2002;87(9):4193-7.

22. Giustina A, Veldhuis JD. Pathophysiology of the neuroregulation of growth hormone secretion in experimental animals and the human. Endocr Rev. 1998;19:717-97.

23. Veldhuis JD, Anderson SM, Iranmanesh A, CyrilYB. Testosterone blunts feedback inhibition of growth hormone secretion by experimentally elevated insulin-like growth factor-l concentrations. J Clin Endocrinol Metab. 2005;90(3):1613-7.

24. Friend KE, Hartman ML, Pezzoli SS, Clasey JL, Thorner MO. Both oral and transdermal estrogen increase growth hormone release in postmenopausal women - A clinical research center study. $J$ Clin Endocrinol Metab. 1996;81:2250-6.

25. Janssen $Y J H$, Helmerhorst F, Frolich M, Roelfsema F. A switch from oral $(2 \mathrm{mg} /$ day) to transdermal ( $50 \mu \mathrm{g} /$ day) $17 \beta$-estradiol therapy increases serum insulin-like growth factor-l levels in recombinant human growth hormone (GH)-substituted women with $\mathrm{GH}$ deficiency. J Clin Endocrinol Metab. 2000;85:464-7.

26. Veldhuis JD, Anderson SM, Kok P, Iranmanesh A, Frystyk J, Orskov $\mathrm{H}$, et al. Estradiol supplementation modulates growth hormone $(\mathrm{GH})$ secretory-burst waveform and recombinant human insulin-like growth factor-l-enforced suppression of endogenously driven $\mathrm{GH}$ release in postmenopausal women. J Clin Endocrinol Metab. 2004;89:1312-8.

27. Guay AT. The emerging link between hypogonadism and metabolic syndrome. J Andrology. 2009;30(4):370-6.

28. Matsumoto AM. Andropause: clinical implications of the decline in serum testosterone levels with aging in men. J Gerontol A Biol Sci Med Sci. 2002;57:M76-99.

29. Zgliczynski S, Ossowski M, Slowinska SJ, Brzezinska A, Zgliczynski W, Soszynski $P$, et al. Effect of testosterone replacement therapy on lipids and lipoproteins in hypogonadal and elderly men. Atherosclerosis. 1996;121(1):35-43.

30. Saad F, Gooren LJ, Haider A, Yassin A. A dose-response study of testosterone on sexual dysfunction and features of the metabolic syndrome using testosterone gel and parenteral testosterone undecanoate. J Androl. 2008;29:102-5.

31. Kapoor D, Goodwin E, Channer KS, Jones TH. Testosterone replacement therapy improves insulin resistance, glycaemic control, visceral adiposity and hypercholesterolaemia in hypogonadal men with type 2 diabetes. Eur J Endocrinol. 2006;154:899-906. 
32. Tan KC, Shiu SW, Janus ED, Lam KS. LDL subfractions in acromegaly: relation to growth hormone and insulin-like growth factor-I. Atherosclerosis. 1997;129:59-65.

33. Arosio M, Sartore G, Rossi CM, Casati G, Faglia G, Manzato E. LDL physical properties, lipoprotein and Lp(a) levels in acromegalic patients. Effects of octreotide therapy. Italian Multicenter Octreotide Study Group. Atherosclerosis. 2000;151:551-7.

34. Roelfsema F, Frolich M, Geelhoed-Duyvestijn PH, Nieuwenhuijzen KAC, Looij BJ. Glucagon-stimulated plasma C-peptide and insulin levels in active and non-active acromegalics. Clin Endocrinol (Oxf). 1985;23:627-34
35. Sonksen PH, Greenwood FC, Ellis JP, Lowy C, Rutherford A, Nabarro JD. Changes of carbohydrate tolerance in acromegaly with progress of the disease and in response to treatment. J Clin Endocrinol Metab. 1967;27:1418-30.

36. Correa LL, Taboada GF, Van Haute FR, Casini AF, Balarini GA, Vieira Neto $L$, et al. Avaliação do metabolismo da glicose em pacientes acromegálicos antes e após tratamento com octreotide LAR. Arq Bras Endrocrinol Metab. 2008;52-61.

37. van der Lely AJ, Hutson RK, Trainer PJ, Besser GM, Barkan AL, Katznelson $\mathrm{L}$, et al. Long-term treatment of acromegaly with pegvisomant, a growth hormone receptor antagonist. Lancet. 2001;358(9295):1754-9. 